\title{
Sagittal Plane Helmet Acceleration at Pole Contact of Alpine Ski Racers is Dependent on Slalom Pole Type and Skill Level
}

\author{
Ronald W. Kipp and John G. Seifert
}

\begin{abstract}
A slalom ski racer's center-of-mass (CoM) has a negative acceleration during a turn. Some of this change in acceleration can be attributed to the physical contact with the pole, and possibly physiological and/or psychological factors of the ski racer. One factor may be the type/size of poles (gates) used to define the race line (route prescribed by competition rules) in slalom. Poles come in a variety of sizes and styles. This study utilized five differing pole types with 12-year-old male and female racers. As a general conclusion, they were faster on the small/shorter poles. They also felt faster, more aggressive, higher level of confidence, and thought their race line was superior on the shorter poles. When looking at the acceleration component, we divided the skiers into "skilled" and "less skilled." The two groups differed on direction of acceleration. The discussion lays out mechanical, physiological, psychological, and technique ramifications to interpret this acceleration paradox.
\end{abstract}

Keywords Ski racers $\bullet$ Slalom $\bullet$ Pole contact $\bullet$ Head injury $\bullet$ Pole design

\section{Introduction}

\subsection{Acceleration}

Alpine ski racers ski around poles which are used to describe the race line. Each ski turn creates a situation where there is a negative acceleration of the skier's centerof-mass (CoM) followed by a period of positive acceleration between turns [1]. To ski fast in slalom, the ski racer skis with their skis close to the pole such that their CoM may actually travel to the inside of the race line. This is a legal passage according to the rules of the Fédération Internationale de Ski (FIS), the governing body of international ski racing. Racers will contact the long pole hitting, blocking, moving,

R.W. Kipp (西)

United States Ski and Snowboard Association, Park City, UT, USA

e-mail: ronwadekipp@gmail.com

J.G. Seifert

Movement Science Laboratory, Montana State University, Bozeman, MT, USA

(C) The Author(s) 2017

I.S. Scher et al. (eds.), Snow Sports Trauma and Safety,

DOI 10.1007/978-3-319-52755-0_11 
or clearing it out of the way with their hand and or ski pole, allowing their body to take a shorter path. This pole contact and movement requires about $181 \mathrm{~J}$ for each pole clear for a World Cup ski racer which may explain part of their CoM-negative acceleration (R. Reid, personal communication, 2010). This negative acceleration would be amplified for young skiers having less mass.

While ski racers must ski around poles, recreational skiers make turns in voluntary locations and tempo. For recreational skiers, turning is an opportunity to manage speed while for the ski racer it is a situation for speed loss to be minimized.

\subsection{Poles and Training Aids}

Poles are used in alpine ski racing to define the race line for the ski racer. The FIS [2] has specifications for pole dimensions used in international competition, while individual countries determine domestic standards.

Poles have a hinge at the snow level. This hinge was developed to keep the pole from becoming dislodged as ski racers skied increasingly closer to the pole eventually discovering they could just mow down the pole with their hand, ski pole, or shin enabling them to take a shorter and therefore faster path. Standards for the hinge are mandated by the FIS.

The FIS has two categories of poles: Type A; which is for use in any FIS race. They have a shaft diameters from 29 to $32 \mathrm{~mm}$. Type B poles $(25-28.9 \mathrm{~mm})$ are for use in any FIS race except World Cup.

Poles vary by their height above the snow surface. All FIS races must employ poles that project a minimum of $1.8 \mathrm{~m}$ above the snow surface [3]. In the United States, U16 competitions must also use the $1.8 \mathrm{~m}$ poles (FIS A or B). However, slalom poles for U14s must be $152 \mathrm{~cm}$ FIS B, while stubby poles are for non-scored events [4].

Young ski racers typically compete with shorter length $(137$ and $152 \mathrm{~cm})$ and smaller diameter poles ( 27 vs. $32 \mathrm{~mm}$ ). In place of poles, props or training aids, such as Whiskers and Heroes, reduce contact while still skiing the intended path. Whiskers are $23 \mathrm{~cm}$ brushes and Heroes are $70 \mathrm{~cm}$ flexible poles. There are no FIS standards for Whiskers and Heroes (SPM S.P.A., Varese, Italy).

The purpose of this study was to determine if pole type or shaft length had an effect on acceleration. Additionally, would finishing time, and subjective feelings of young ski racers be altered by pole length?

\section{Methods}

\subsection{Subjects}

Eighty seven (54 females, 33 males) club level skiers from three different USSA (United States Ski and Snowboard Association) affiliate ski teams participated in the study. Data are presented as average ( \pm standard deviation). Average age was 
12 years 10 days $( \pm 1.0$ year). Skiers reported an average $4.6( \pm 0.9)$ years of race experience. Average height was $153.4( \pm 8.9) \mathrm{cm}$, average weight $41.05( \pm 10.88) \mathrm{kg}$, and average ski length was $140.4( \pm 7.80) \mathrm{cm}$. "Skilled" and "unskilled skiers" were defined by the nine skiers with the fastest and slowest run time. This was then confirmed with video by a USSA-certified ski coach.

\subsection{Courses}

The study was conducted on 12 different slopes at Bridger Bowl Ski Area, Montana, and Park City Mountain Resort, Utah, over 11 separate days in winter conditions. The 12 slopes averaged 20.9 degrees $( \pm 2.7)$. They were relatively even in pitch with minimal side hill. Individual average pitches consisted of 20, 23.5, 28, 22, 22, 18 , $18,20,20,19.4,20,20^{\circ}$.

An identical ten gate slalom course was set for all trials. It consisted of four open gates set at $9 \mathrm{~m}$ (gate to gate) to a three gate flush at $5.5 \mathrm{~m}$ followed by three open gates set at $9 \mathrm{~m}$ (gate to gate) to the finish $9 \mathrm{~m}$ away (see Fig. 1). Offset was $4 \mathrm{~m}$ with the flush in line with the same $4 \mathrm{~m}$ horizontal distance. All courses were slipped frequently in order to minimize rut development and overall course deterioration.

Skiers completed one or more runs on each pole type course in a random order. Multiple runs on the same course were used to check for reliability between runs.

Fig. 1 Course set-up used for all data collection

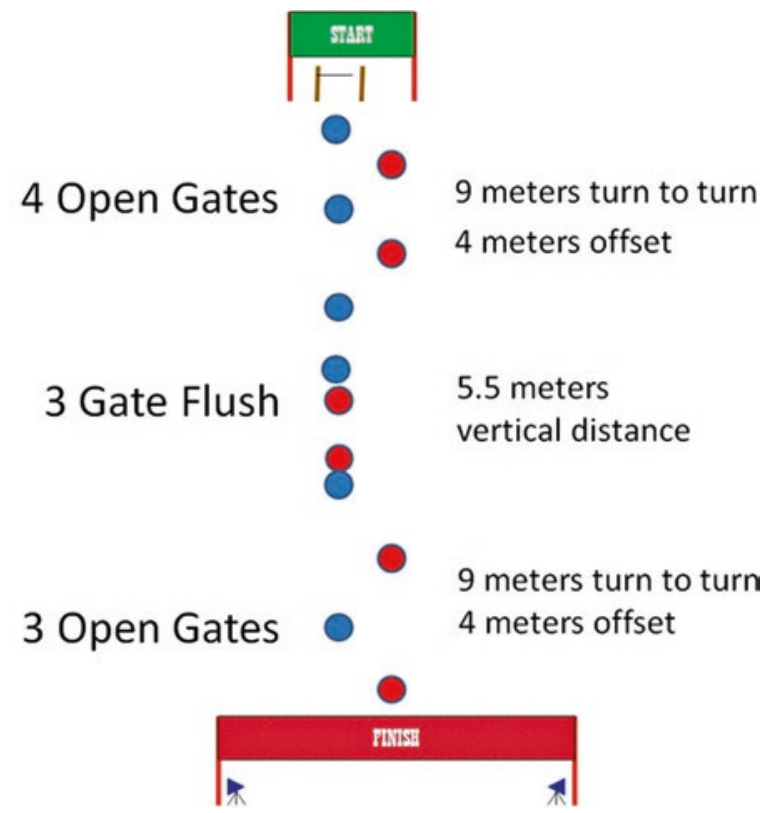




\subsubsection{Pole Type Used During Each Testing}

- Whiskers: Bright orange and green plastic were alternated in the courses. $23 \mathrm{~cm}$ length, with a lightly threaded $20.5 \mathrm{~cm}$ base.

- Hero: Red and blue, pliable, rubber-like shaft, $35 \mathrm{~mm}$ in diameter and $70 \mathrm{~cm}$ long. $86 \mathrm{~cm}$ overall length including BrushGrip ${ }^{\mathrm{TM}}$ Base.

- $137 \mathrm{~cm}: 27 \mathrm{~mm}$ diameter shaft that is $137 \mathrm{~cm}$ off the snow with BrushGrip ${ }^{\mathrm{TM}}$ Base.

- $152 \mathrm{~cm}: 27 \mathrm{~mm}$ diameter shaft that is $152 \mathrm{~cm}$ off the snow with BrushGrip ${ }^{\mathrm{TM}}$ Base.

- $180 \mathrm{~cm}$ : $27 \mathrm{~mm}$ diameter shaft that is $180 \mathrm{~cm}$ off the snow with BrushGrip ${ }^{\mathrm{TM}}$ Base.

All poles, Heroes, and Whiskers were manufactured by SPM and were new at the beginning of the study. Pole hinges showed no sign of deterioration at the end of the study.

\subsubsection{Timing}

The order in which the courses were skied was randomized for each testing bout.

Timing was with a Brower XS Timing System (Salt Lake City, UT, USA).

\subsubsection{Acceleration}

A gyroscopically controlled three-dimensional accelerometer (Electronic Realization, Bozeman, MT) was attached to the crown of the skier's helmet. Sagittal plane, the fore/aft direction was defined as " $x$ " the vertical direction as " $y$ " the lateral direction as " $\mathrm{z}$ ".

\subsubsection{Skier Perception}

Skier perception immediately at the conclusion of each run was collected. The subject responded to four statements indicating their perception about speed, aggressiveness, confidence, and line with a 10-point Likert scale.

On that course I felt:

- Slow...fast

- Not aggressive...very aggressive

- Not confident...very confident

- Not a good line... a very good line

The higher the response number, the more positive the skier felt. A single factor ANOVA and post hoc Tukey HSD were used to calculate significance. 


\section{Results}

\subsection{Time}

For the analysis 522 valid runs were used.

Average run time and standard deviation (SD) in seconds per pole type (Table 1):

Statistically (single factor ANOVA and post hoc Tukey HSD):

- Whiskers and Heroes times are not different from each other.

- Whiskers and Hero times are different from $137 \mathrm{~cm}, 152 \mathrm{~cm}, 180 \mathrm{~cm}(p=0.01)$.

- $137 \mathrm{~cm}$ and $152 \mathrm{~cm}$ times are not different from each other.

- $137 \mathrm{~cm}$ and $152 \mathrm{~cm}$ times are different from Whiskers, Heroes $(p=0.01), 180 \mathrm{~cm}$ $(p=0.01, p=0.05)$.

- $180 \mathrm{~cm}$ times are different from all other poles $(p=0.01), 137 \mathrm{~cm}(p=0.05)$.

\subsubsection{Reliability Between Runs of Same Pole Type (Within Skier)}

Determining the variability between run times was important to establish test-retest reliability. 141 duplicate runs from the five pole/training aids were used to determine reliability. One factor to be noted was that the runs used were all from the same measurement day. Due to the multivariate nature of ski racing, this was the best option to control for the many variables that would most likely change from day to day.

A very high reliability [Pearson Product-Moment Correlation coefficient (r)] was found for all pole and training aid types (Table 2):

Table 1 Average run time \pm SD (s) per pole or training aid type

\begin{tabular}{l|l|l}
\hline & Average & SD \\
\hline Whiskers & 11.810 & 1.652 \\
\hline Hero & 11.769 & 1.981 \\
\hline $137 \mathrm{~cm}$ & 12.644 & 1.772 \\
\hline $152 \mathrm{~cm}$ & 12.502 & 1.600 \\
\hline $180 \mathrm{~cm}$ & 13.011 & 1.770 \\
\hline
\end{tabular}

Table 2 Reliability for each pole and training aid type

\begin{tabular}{l|l}
\hline & $r$ \\
\hline Whiskers & 0.953 \\
\hline Hero & 0.991 \\
\hline $137 \mathrm{~cm}$ & 0.978 \\
\hline $152 \mathrm{~cm}$ & 0.956 \\
\hline $180 \mathrm{~cm}$ & 0.948 \\
\hline Average & 0.967 \\
\hline
\end{tabular}




\subsection{Perception}

Skiers showed consistent trends in perception after their conclusion.

\subsubsection{Questionnaire Results (Table 3)}

\subsection{Acceleration}

\subsubsection{Mean Acceleration Skilled vs. Less Skilled}

There were significant differences for mean sagittal plane helmet acceleration between the three pole types and the two training aids. Also, a significant difference was observed when the skiers were grouped into "skilled" and "less skilled" cohorts $(p=0.0000)$. The skilled skiers having a positive acceleration while the less skilled a negative acceleration. See Fig. 2.

Table 3 Results from the 0-10-point Likert scales asked at the conclusion of each run

\begin{tabular}{l|l|l|l|l}
\hline & Slow/fast & Aggressiveness & Confidence & Line \\
\hline Whiskers & 8.04 & 8.25 & 8.46 & 7.90 \\
\hline Hero & 7.85 & $7.97^{\mathrm{a}}$ & 8.57 & 7.80 \\
\hline $137 \mathrm{~cm}$ & 7.80 & 7.99 & 8.49 & 7.92 \\
\hline $152 \mathrm{~cm}$ & 7.97 & 8.06 & $8.15^{\mathrm{b}}$ & $7.54^{\mathrm{c}}$ \\
\hline $180 \mathrm{~cm}$ & $6.99^{\mathrm{d}}$ & $7.31^{\mathrm{d}}$ & $7.58^{\mathrm{d}}$ & $6.91^{\mathrm{d}}$ \\
\hline
\end{tabular}

${ }^{\text {aS }}$ Significantly different from Whiskers $(p=0.05)$

${ }^{\mathrm{b}}$ Significantly different from $137 \mathrm{~cm}$ and Heroes $(p=0.01)$

${ }^{\mathrm{c}}$ Significantly different from $137 \mathrm{~cm}(p=0.01)$ and Whiskers $(p=0.05)$

${ }^{\mathrm{d}}$ Significantly different from all other poles and training aids $(p=0.01)$

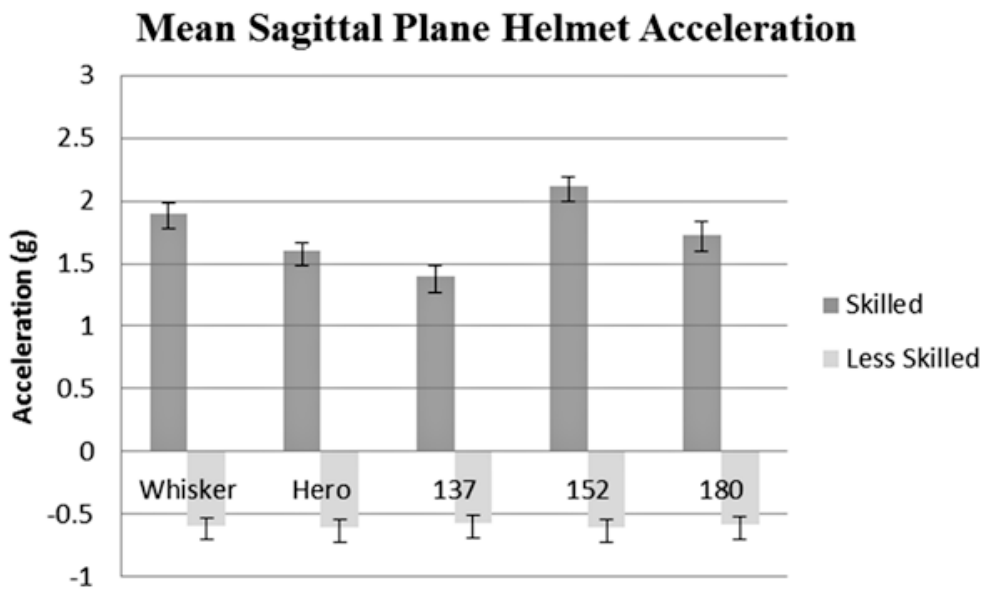

Fig. 2 Helmet acceleration across pole type 
There was also a significant difference in mean acceleration between the skilled and less skilled in the vertical $(p=0.000)$ and lateral $(p=0.000)$ directions.

There were no significant differences in mean acceleration in any of the three directions between the five pole types, nor was there any difference when grouped into the two training aids (Whisker and Hero) and the two longer pole lengths (152 and $180 \mathrm{~cm}$ ) for any of the three directions. See Fig. 3.

\section{Mean Acceleration Skilled Skiers}

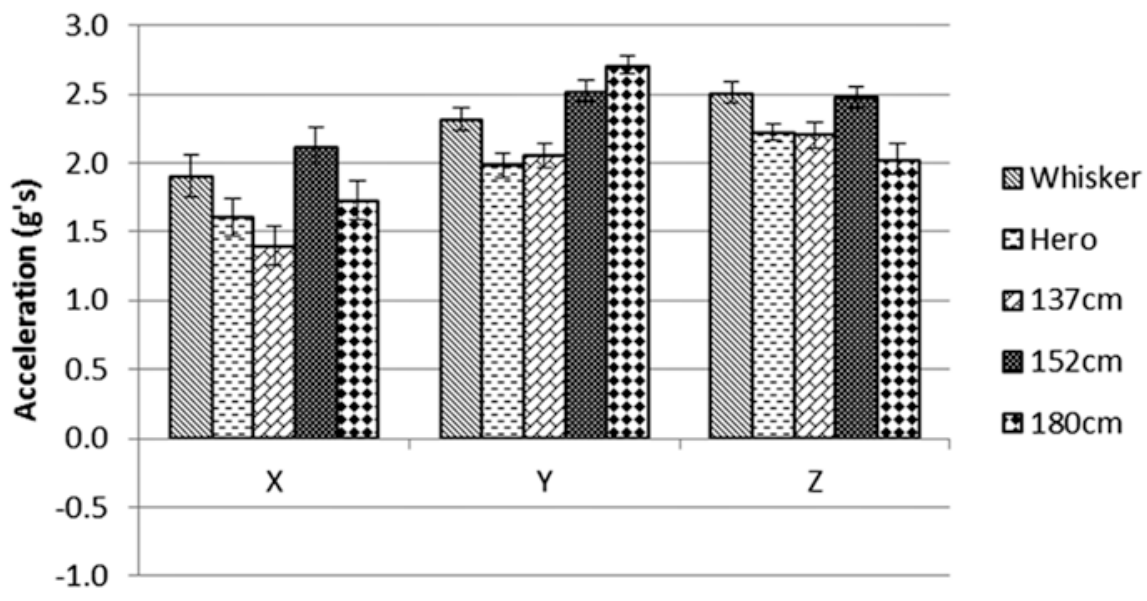

\section{Mean Acceleration Less Skilled Skiers}

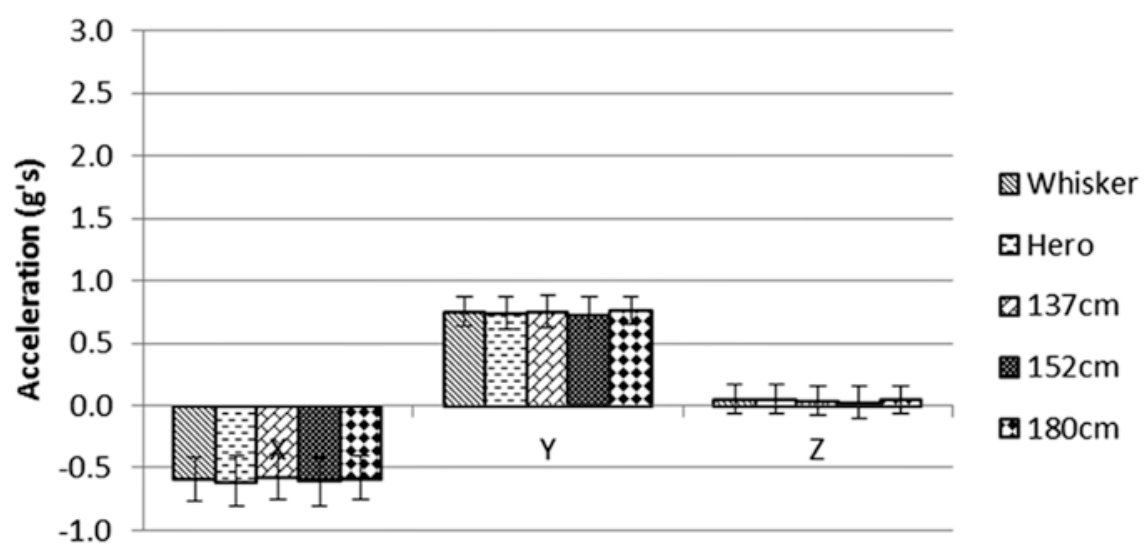

Fig. 3 Mean accelerations in the fore/aft (x), vertical (y), and lateral (z) direction for the three pole lengths and two training aids for skilled and less skilled ski racers 


\subsection{Peak Acceleration}

When averaged over ten consecutive turns there was significant differences for peak acceleration for the skilled group in the $\mathrm{z}$ direction $(p=0.03)$ when the short props were compared to the two long poles, and for the less skilled in the y direction $(p=0.05)$. When the short props were compared to the two long poles, all other short props vs. tall poles were nonsignificant.

\section{Discussion}

Three pole types (lengths) and two training aids were selected for this study. The $27 \mathrm{~mm}$ shaft diameter was chosen over the $25 \mathrm{~mm}$ shaft based upon a previous USSA club questionnaire and US supplier recommendations. The 137 and $152 \mathrm{~cm}$ lengths were chosen as they are popular lengths used in the United States for young ski racers, and $180 \mathrm{~cm}$ as the FIS standard. Whiskers and Heroes were included as they are popular training choices.

The subjects were very homogenous in age. The control of age was important to eliminate or reduce age as an extraneous variable that could potentially affect the results. The study was conducted over 11 nonconsecutive days on 12 differing slopes. This helped to ensure that the results would be representative to the population in question.

Acceleration was positive for the skilled skiers and negative for the less skilled skiers across all three pole lengths and the two training aids. The question must be asked, was this difference in acceleration due to the pole length or type, or was it indicative of the skilled behavior?

Did pole type effect time? Whisker and Heroes were the fastest, with the 137 and $152 \mathrm{~cm}$ next, followed by $180 \mathrm{~cm}$ as the slowest. This time transition from shorter pole to longer pole agrees with intuitive logic and could be based on the mechanical energy dissipation consequence of shaft deformation, shaft acceleration, and hinge resistance, or possibly the influence of pole size on the racer's psychological state.

The run time of Whiskers and Heroes were not statistically different from one another. Contact with the pole or any line alteration with the Hero did not seem to influence the finish time when compared to the Whiskers. This was confirmed with the skier's subjective impression giving a similar response (Whisker: 8.04, Hero: 7.85) when asked if they felt "slow" (0) or "very fast" (10). The skiers also report feeling less aggressive on the Hero (7.97) courses than the Whisker (8.25) courses $(p=0.01)$.

The 137 and $152 \mathrm{~cm}$ poles were not significantly different from each other in time. However, they were significantly different from the Whisker, Hero, and $180 \mathrm{~cm}$. While the skier's time was not statistically different, they did report feeling less confident and less satisfied with their line when skiing the $152 \mathrm{~cm}$ pole.

The $180 \mathrm{~cm}$ poles elicited the slowest time, which was also the feeling generated from the skiers. The skiers also felt less aggressive and less confident along with less satisfaction with their line. 
A very high reliability was found for all five pole types (cumulatively $\underline{r}=0.967$ ). Reliability is an essential component of validity which was essential for this study. Being assured that a run time per pole type was indeed indicative of that pole type was crucial to the interpretation of the results.

\subsection{Possible Explanations}

There are natural acceleration consequences of a skier center-of-mass during a ski turn. Reid and coworkers have used kinematic data with elite ski racers showing that during a slalom turn, the skier's CoM goes through a period of negative acceleration that is followed by a period of positive acceleration between turns [1]. The period of negative acceleration starts when the turn is initiated which is before flex pole contact. Recently, Schindelwig and coworkers used finite element analysis to demonstrate the increase in race time due to a longer flex pole in children's slalom racing [5].

\subsubsection{Physical Contact with the Pole}

It has been calculated that elite ski racers contacting an FIS A pole will use $45 \mathrm{~J}$ for work to deform the pole, $108 \mathrm{~J}$ to accelerate the pole, and $28 \mathrm{~J}$ to overcome hinge resistance for $181 \mathrm{~J}$ of work. This comes out to be about $4 \%$ of his kinetic energy (R. Reid, personal communication, 2010). Upon video analysis, the less skilled skiers did not necessarily contact the pole.

\subsubsection{Anticipatory Postural Control}

Racers contact the pole by hitting, blocking, moving, or clearing it out of the way with their hand or ski pole allowing their body to take a shorter path. The action to perform this "clearing" maneuver involves raising the arm which is accomplished with the pectoralis major and anterior fibers of the deltoid. While this is the mechanism, a phenomena observed and described by Belen'kii et al. [6] that standing subjects when asked to raise their arm, utilized both their prime movers (arm) and their postural muscles (trunk and legs). The postural muscles innervate $50 \mathrm{~ms}$ prior to the prime mover activation. This was in anticipation the upcoming destabilizing effects of the arm movement.

The more skilled ski racers could be anticipating the impact of pole contact with their postural muscles. These could be with the lower body or the neck muscles. In such a case, they are accelerating their upper body in proactive manner intuitively knowing that the pole will have a negative accelerating effect on them. This anticipatory postural adjustment has been shown to be a learned response by Cordo and Nashner [7]. The less skilled ski racers may not have had ample experience to have sufficiently learned this anticipatory postural mechanism and partially explain the resultant negative acceleration seen in this study. 


\subsubsection{Vestibulo-Ocular Reflex}

The vestibulo-ocular reflex (VOR) maintains the visual image during linear and angular head movement. While skiing skill may be thought of as the ability to manipulate the skis in an efficient strategy, there may be an adaptive mechanisms in the central nervous system that is important in maintaining the VOR [8, 9]. This adaptation may be a result of increasing skiing skill or possibly a manifestation of maturity.

VOR motor learning functions to improve motor performance. This occurs via the gain of the VOR or the ratio of eye to head speed and its phase which is the temporal relationship between eye and head movement. Eye movements are opposite in direction and approximately equal in speed to the head movement [10]. It could be postulated that the positive acceleration from the skilled skiers at pole contact was an anticipatory learned response knowing that the pole would provide some negative acceleration at contact.

\subsubsection{Psychological Factors}

One explanation for the positive and negative acceleration at pole contact from the skilled and less skilled can be explained by the novelty of situation. The skilled skier has encountered this situation many times and knows what to expect. The less skilled skier is not as sure what to expect. The less skilled skiers' negative helmet acceleration may be a result of moving away from the pole in a ducking or avoidance response.

\subsubsection{Ski Pressure Management}

Elite ski racers strive to pressure their skis while they are in the fall-line or in the part of the turn that is pointed down the hill. Less skilled ski racers and recreational skiers that are striving for speed control as opposed to speed conservation will have pressure later in the turn. This type of turn will not accelerate in the turn but constantly show a negative acceleration throughout the turn.

\subsubsection{Movement Decision Under Risk}

Alpine skiing can be perceived as a risky endeavor. The skier skill level can lead to behavior that is based on risk avoidance or risk-seeking objectives [11, 12]. A risk avoidance situation can be postulated from the less skilled ski racers that will have greater variability in most aspects of their skiing technique suggesting increased instability [13]. Some of the negative acceleration seen in this study could be explicated by the risk perception that occurs during the middle of the turn when the skis are pointed at their most down the hill attitude and coinciding with a possible pole contact. Both could be assumed to be a "risky" moment in time. 


\subsection{What Does It Mean for Safety?}

There are many ways to become injured skiing. The most obvious is losing some level of control of the skis. When injury does happen, there is a $50 \%$ chance it is to the lower extremity, which today usually means an ACL injury [14, 15]. Most of the ACL injuries among recreational skiers are reported to occur during falling $[16,17]$. The three mechanisms of ACL injury all involve falling either in a backward or a forward direction. [18]. Specifically, the "phantom-foot," "boot-induced anterior drawer," "slip-catch," "landing back-weighted," and the "dynamic snowplow" mechanisms all have the skier falling backwards while the "valgus-external rotation" the skier falls forward. This is different from the mechanisms for other sports, such as American football, basketball, and soccer. In these activities, the foot is in firm contact with the ground and usually results in deceleration, change-of-direction, or some sort of direct blow. In skiing, the skis are constantly sliding forward while not in contact with the ground. This forward motion constantly challenges the skier to maintain control of their fore/aft relationship.

Fore/aft balance has always been a nemesis to the skier. The importance to recreational skiers is that the Professional Ski Instructor's of American lists "controlling the relationship of the center-of-mass to the base of support to direct pressure along the length of the skis" as one of their five Fundamentals of Skiing [19]. And among elite ski racers, fore/aft movement has been found to be correlated with speed in a race course $[20,21]$.

While it would be inappropriate to suggest that having superior fore/aft balance would lead to greater control, and therefore greater safety, it would conversely be irresponsible to overlook the relevancy and connection between sagittal plane control and the possible association with safety.

Acknowledgements World Cup Supply, VT, USA for supplying new SPM poles, Whiskers, and Heroes. Skiers and coaches from the Park City Ski Team, Summit Ski Team, and Bridger Ski Foundation for their help and participation.

\section{References}

1. Reid R, Gilgien M, Moger T et al (2009) Turn characteristics and energy dissipation in slalom. In: Müller E et al (eds) Science and Skiing IV. Meyer \& Meyer Sport, Oxford, pp 419-429

2. FIS international ski competition rules book IV joint regulations for alpine skiing (2016) International Ski Federation FIS. Oberhofen, Switzerland

3. The International Ski Competition Rules (ICR) (2012) International Ski Federation FIS. Oberhofen, Switzerland

4. 2016 USSA Alpine Competition Guide (2015) U. S. Ski and Snowboard Association. Park City, UT

5. Schindelwig K, Kaps P, Nachbauer W (2015) Short and thin walled flex poles for children slalom race. In: Petrone N, Marcolin G (eds) 21st International Congress on Ski Trauma and Skiing Safety Book of Abstract, p 97 
6. Belen'kii VY, Gurfinkel VS, Paltsev YI (1967) Elements of control of voluntary movements. Biofizika 12:135-141

7. Cordo P, Nashner L (1982) Properties of postural adjustments associated with rapid arm movements. J Neurophysiol 47:287-302

8. Page GD, Telford L, Seidman SH et al (1998) Human vestibuloocular reflex and its interactions with vision and fixation distance during linear and angular head movement. J Neurophysiol 80:2391-2404

9. Peterka RJ, Black FO, Schoenhoff MB (1990) Age-related changes in human vestibulo-ocular reflexes: sinusoidal rotation and caloric tests. J Vestib Res 1(1):49-59

10. Broussard DM, Kassardjian CD (2004) Learning in a simple motor system. Learn Mem $11: 127-136$

11. O’Brien MK, Ahmed AA (2016) Rationality in human movement. Exerc Sport Sci Rev 44(1):20-28

12. Pienciak-Siewert A, Barletta AJ, Ahmed AA (2013) Transfer of postural adaptation depends on context of prior exposure. J Neurophysiol 111:1466-1478

13. Manista GC, Ahmed AA (2012) Stability limits modulate whole-body learning. J Neurophysiol 107:1952-1961

14. Ekeland A, Rødven A (2009) Injury trends in Norwegian ski resorts in the 10 year period 1996-2006. In: Johnson RJ (ed) Skiing trauma and safety: 17th volume, ASTM STP 1510. American Society for Testing and Materials, West Conshohocken, PA, pp 31-38

15. Johnson RJ, Ettlinger CF, Shealy JE (2009) Updates on injury trends in alpine skiing. In: Johnson RJ, Shealy JE, Langran M (eds) Skiing trauma and safety: 17th volume. American Society for Testing and Materials, West Conshohocken, PA, pp 11-22

16. Fischer JF, Leyvraz PF, Bally A (1994) A dynamic analysis of knee ligament injuries in alpine skiing. Acta Orthop Belg 60:194-203

17. Ruedl G, Linortner I, Schranz A et al (2009) Distribution of injury mechanisms and related factors in ACL-injured female carving skiers. Knee Surg Sports Traumatol Arthrosc 17:1393-1398

18. Bare T (2011) Mechanisms of anterior cruciate ligament injury in world cup alpine skiing: a systematic video analysis of 20 cases. Am J Sports Med 39:1421-1429

19. PSIA Alpine Certification Standards (2014) ASEA and ASEA-EF. Lakewood CO

20. Kipp RW, Reid RC, Gilgien M et al (2008) Slalom performance in élite alpine ski racing can be predicted by fore/aft movement dynamics. Med Sci Sports Exerc 40(5):S1246

21. Kipp RW, Reid RC, Gilgien M et al (2009) Fore/aft movement is adapted to gate separation by elite alpine racers in slalom. In: 14th Annual Congress of the European College of Sports Science Book of Abstracts. Oslo, Norway, p 171

Open Access This chapter is distributed under the terms of the Creative Commons AttributionNoncommercial 2.5 License (http://creativecommons.org/licenses/by-nc/2.5/) which permits any noncommercial use, distribution, and reproduction in any medium, provided the original author(s) and source are credited.

The images or other third party material in this chapter are included in the work's Creative Commons license, unless indicated otherwise in the credit line; if such material is not included in the work's Creative Commons license and the respective action is not permitted by statutory regulation, users will need to obtain permission from the license holder to duplicate, adapt or reproduce the material. 